\title{
NOVEL LEARNING STRATEGY BASED ON GENETIC PROGRAMMING FOR CREDIT CARD FRAUD DETECTION IN BIG DATA
}

\author{
Ibtissam Benchaji, Samira Douzi and Bouabid El Ouahidi \\ IPSS. Faculty of Sciences, University Mohammed V, Rabat, Morocco
}

\begin{abstract}
Due to the growing volume of monetary transactions on the internet, credit card fraud poses many challenging issues for banks and financial institutions, thus forcing them to continuously improve their fraud detection systems. However, current fraud detection techniques are far from accurate and fail to minimize the false alarm rates, which can cause inconvenience and dissatisfaction for customers in e-banking system. Furthermore, there are several factors that decrease the performance of Fraud Detection Systems (FDS), such as skewed distribution, concept drift, supports real time detection, large amount of data etc. In this paper, we address the highly imbalanced class issue faced by a FDS when working with big data and propose a novel method of generation of data set's minority class based on K-means clustering method and genetic algorithm to improve classification performance in credit card fraud detection. In our experiments, we apply the proposed approach to synthetic unbalanced credit card fraud data set to demonstrate its effectiveness by means of the most appropriate performance measures for fraud detection purposes.
\end{abstract}

\section{KEYWORDS}

Big Data, Fraud Detection, Imbalanced Dataset, K-means Clustering, Genetic Programming

\section{INTRODUCTION}

As the improvement of technology and the advent of new payment solutions has brought many new benefits to the society and established credit card as the most popular payment mode for both online and offline purchases, it has also resulted unfortunately in an increase of card fraud activities.

Automatic credit card fraud detection is a challenging machine learning problem and different techniques have been proposed to deal with it (Aleskerov, E. et al, 1997; Whitrow, C. et al, 2009; Sánchez, D. et al, 2009; Bhattacharyya, S. et al, 2011; Jha, S. et al, 2012; Sahin, Y. et al, 2013; Dal Pozzolo, A. et al, 2014; Bahnsen, A. C. et al, 2015). However, several aspects must be considered at the same time when building a system for card fraud detection. For instance, the data distribution evolves over time because of seasonality and new attack strategies. On the other hand, the system has to respond in very short times to become useful in real scenarios. Another critical aspect is the skewness of the data since legitimate transactions far outnumber the fraudulent ones. In fact, Class distribution is extremely unbalanced in credit card transactions, because frauds are typically less than 1\% of the overall transactions, as shown in (Dal Pozzolo, A. et al, 2014 ; Krivko, M., 2010). Such imbalanced sets require additional precautions because the prediction accuracy of standard machine learning techniques, especially for the minority class which is the class of interest, tends to be lower. Thus, misclassifying the minority class has much higher cost compared to misclassifying a majority class instance. Notice that, when the system predicts a transaction as fraudulent when in fact it is not (false positive), the financial institution has an administrative cost, as well as a decrease in customer satisfaction. On the contrary, when the system does not detect a fraudulent transaction (false negative), the amount of that transaction is lost.

In this paper, we address primarily the last issue and propose the generation method of imbalanced data set's minority class, by using K-means clustering method and genetic algorithm, thus new mimicked minority class samples can be obtained through crossover on the basis of cluster, which are then merged with training data into an augmented training set so that the effectiveness of a classifier can be improved. We provide the obtained results to support and validate our findings. 
This work is organized as follows. In Section 2, related work is analyzed and discussed. In Section 3, we present the organization of our proposed methodology. Section 4 describes the dataset used in this work and discusses the obtained results. Finally, Section 5 concludes the paper and proposes ideas for future research.

\section{RELATED WORK}

As introduced, the major challenge to be addressed when designing a Fraud Detection System is handling the class imbalance. A dataset is called imbalanced when the number of negative (majority) instances outnumbers the amount of positive (minority) class instances. Figure 1 illustrates the class distribution of Credit Card Dataset used in our experiments.

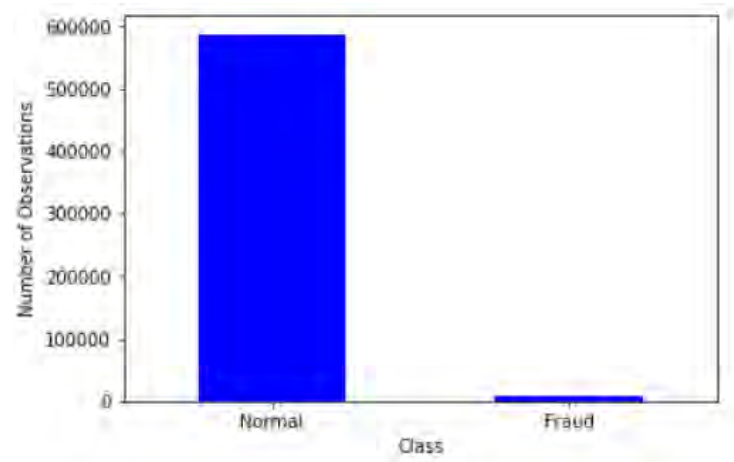

Figure 1. Class distribution of Credit Card Data Set

Hence, standard machine learning algorithms that maximize overall accuracy tend to classify all observations as majority class instances and perform poorly in terms of predictive accuracy on the minority class (low recall). In real world applications, classification accuracy of the smaller class is critically important because the minority class usually is the class of great interest such as cases of fraud. Thus, misclassifying the minority class has much higher cost compared to misclassifying a majority class instance.

In this regard, various approaches have been proposed to address the imbalanced datasets issue and improve the performance of predictive modeling. These approaches could be mainly divided into two categories. The first category solves the problem at data level, that is, data resample techniques are performed to directly alter the original dataset which is not balanced by removing samples from the majority class (undersampling) or replicating training samples of the minority class (oversampling) (Dal Pozzolo, A. et al, 2015). Advanced oversampling methods like SMOTE (Chawla, N. et al, 2002) generate synthetic training instances from the minority class by interpolation, instead of sample replication. However, these techniques present significant drawbacks, such as undersampling may lose some potential information, and oversampling may lead to overfitting.

The second main type of approaches that considers classification of imbalanced data sets at algorithm level is known as Cost based learning or Ensemble learning (Cieslak, D. A. et al, 2012). Ensemble methods include bagging and boosting strategies that aim to combine several weak classifiers as one strong classifier which can improve the classification performance of imbalanced dataset. The ideas of Bagging (Bootstrap Aggregating) (Breiman, L., 1996) is to split the majority class into multiple sub sets, and for each sub set, training a basic weak classifier together with the minority class, then integrate these classifiers into a strong classifier. The AdaBoost (Adaptive Boosting) (Freund, Y. and Schapire, R. E., 1996) talks about the misclassified samples that generated by the former basic classifier will be augmented by assigning weights. The weighted data sets will be send to next basic classifier to reduce the total bias error. AdaBoost is equivalent to resampling the data space (Sun, Y. et al, 2007), which are applicable to most classification systems without changing their learning methods. Besides, it could eliminate the extra learning cost for exploring the optimal class distribution and representative samples (Ali, A. et al, 2015). Moreover, compared with the method of eliminating samples from data set, it reduces the information loss, overfitting risk and bias error of a certain classification learning method (He, H. and Garcia, E. A., 2009). 
Instead of these approaches, the present paper focuses on generating new samples for minority class with respect to the original set, using clustering and genetic operators, which is a novel approach of oversampling the minority fraudulent data that aims to improve the performance of imbalanced data classification when designing an effective Fraud Detection System.

\section{PROPOSED METHODOLOGY}

In this paper we propose a new oversampling approach for improving classification effectiveness in credit card fraud detection based on k-means cluster and genetic algorithms. Firstly, we apply the k-means algorithm to split minority class of fraud instances into clusters according to their similarities. The goal of this clustering method is to have in each cluster samples that are as much similar as possible which can guarantee that new samples of whole minority class have better coverage and representation. Thus, by means of crossover and mutation operators we generate in each cluster new synthetic minority class instances that mimic the original minority class examples as closely as possible and merge them with the original dataset into an augmented training set. Figure 2 shows the big picture of the proposed method.

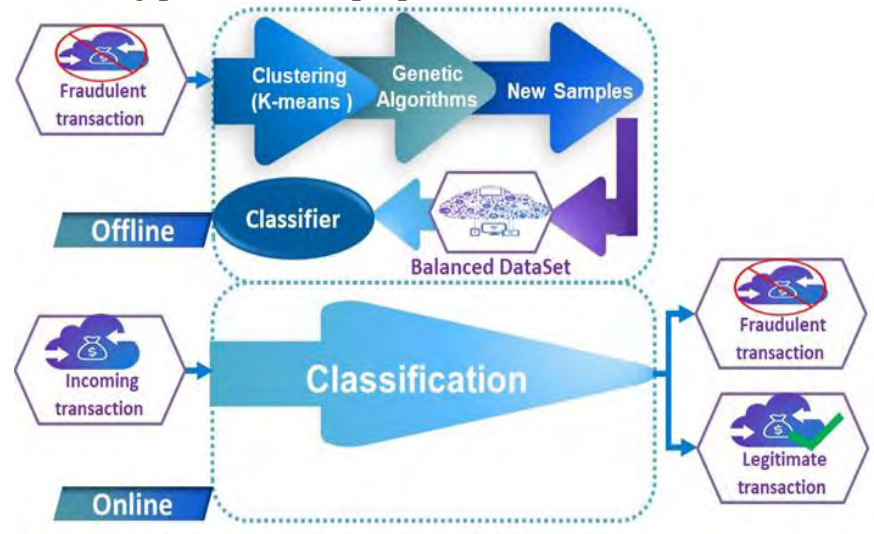

Figure 2. The illustration of credit card fraud detection system

\subsection{K-means Clustering Method}

Clustering is a process of partitioning a set of samples into a number of groups without any supervised training. The goal of this technique is to categorize the records of a dataset in such a way that similar records are grouped together in a cluster and dissimilar records are placed in different clusters. The most popular clustering algorithm is k-means because of its simplicity and efficiency (Zhang, J. et al, 2013; Jain, A.K, 2010).

$\mathrm{K}$-means algorithm groups $\mathrm{N}$ objects into $\mathrm{K}$ clusters maintaining high intra group similarity and low inter group similarity of the objects. The K-means algorithm places a data object into a group by finding closest cluster center using a distance measure. This measure calculates similarity distance between each data objects and centroids. Among many available distance measures, Euclidean distance is the widely used measure for distance calculation between objects and centroids (Bawane, V. S. and Kale, S. M., 2015).

Given a set of $\mathrm{n}$ data points $\mathrm{D}=\left\{x_{1}, x_{2}, x_{3}, \ldots, x_{n}\right\}$ in $R^{d}$ and an integer $\mathrm{K}$, the $\mathrm{K}$-means problem is to determine a set of $\mathrm{K}$ centroids $\mathrm{C}=\left\{\boldsymbol{C}_{1}, \ldots, \boldsymbol{C}_{k}\right\}$ in $R^{d}$ so as to minimize the following error function :

$$
J=\sum_{j=1}^{k} \sum_{i=1}^{n}\left\|x_{i}^{(j)}-c_{j}\right\|^{2}
$$

The K-Means Clustering algorithm is composed of the following steps:

1. Select $\mathrm{k}$ points at random as cluster centers. 
2. Assign objects to their closest cluster center according to the Euclidean distance function.

3. Calculate the centroid or mean of all objects in each cluster.

4. Repeat steps 2, 3 and 4 until the same points are assigned to each cluster in consecutive rounds.

As K-Means algorithm requires the number of clusters as input, we will use the elbow method to get the optimal number of clusters. The idea of the elbow method is to run k-means clustering on the dataset for a range of $\mathrm{K}$ values and for each value of $\mathrm{K}$ calculates the mean distance between data points and their cluster centroid. Since increasing the number of clusters will always reduce the distance to data points, increasing $\mathrm{K}$ will always decrease this metric, to the extreme of reaching zero when $\mathrm{K}$ is the same as the number of data points. Thus, the elbow method plots the mean distance to the centroid as a function of $\mathrm{K}$ and the "elbow point," where the rate of decrease sharply shifts, can be used to roughly determine K, as depicted in Figure 3.

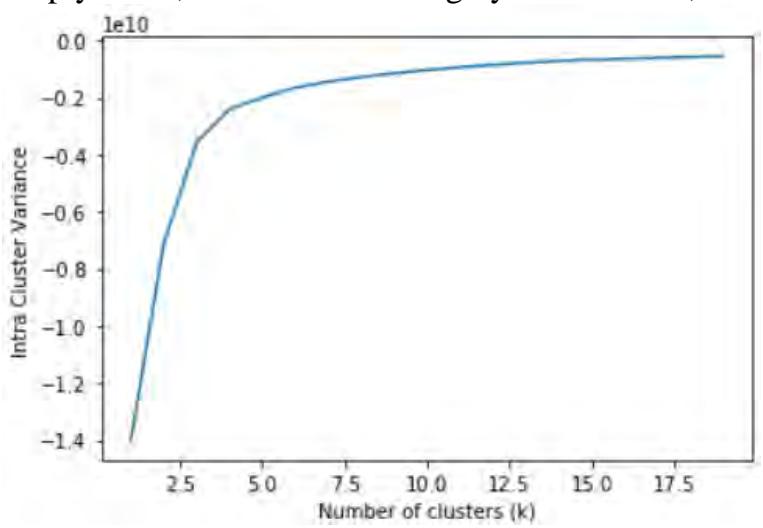

Figure 3. Elbow Method for selection of optimal "K" clusters applied to our DataSet

In Figure 3, it can be observed that an elbow point occurs at $K=4$. Hence, for values larger than $K=4$, the difference in SSE (Sum of Squared Errors) starts to decrease and diminishes gradually. Hence, we will choose to have 4 clusters and provide this value as input to the K-Means algorithm.

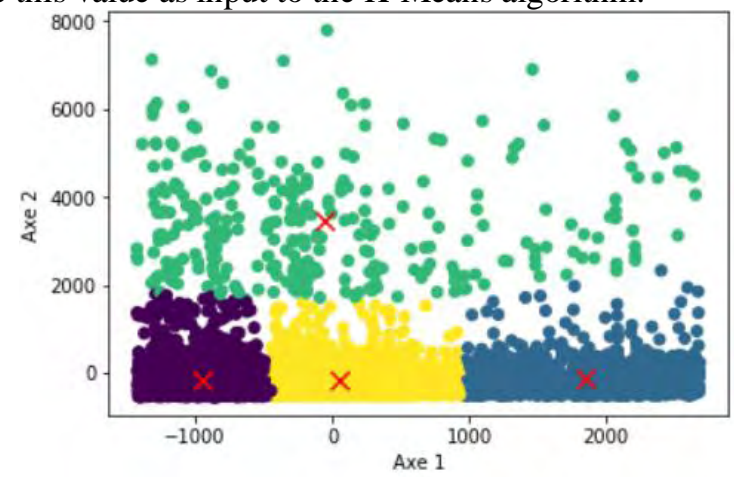

Figure 4. Fraud Data repartition using K-Means Algorithm

In the proposed paper, K-Means Clustering is applied on the dataset taken for study to split minority class of fraud instances into 4 clusters according to their similarities. New mimicked samples can be created from samples that are as much similar as possible in each cluster through genetic operators. Thus, every cluster can obtain certain proportion of new samples, which can guarantee that new samples of whole minority class have better coverage and representation. Figure 4 presents the scatter plot of the 4 clusters formed.

\subsection{Genetic Algorithms}

Genetic algorithms were inspired from Darwin's theory of evolution and were pioneered by John Holland (Holland, J. H., 1992). A genetic algorithm can be defined as a search algorithm based on the mechanics of 
natural selection and natural genetics (Goldberg, D., 1983). Genetic algorithms have at least the following elements in common: Populations of chromosomes, selection according to fitness, crossover to produce offspring, and random mutation of a new offspring (Ben Amor, H. and Rettinger, A., 2005). In a broader usage of the term, a genetic algorithm is any population-based model that uses selection and recombination operators to generate new sample points in a search space. Figure 5 and Figure 6 depicts a schematic view of different steps involved.

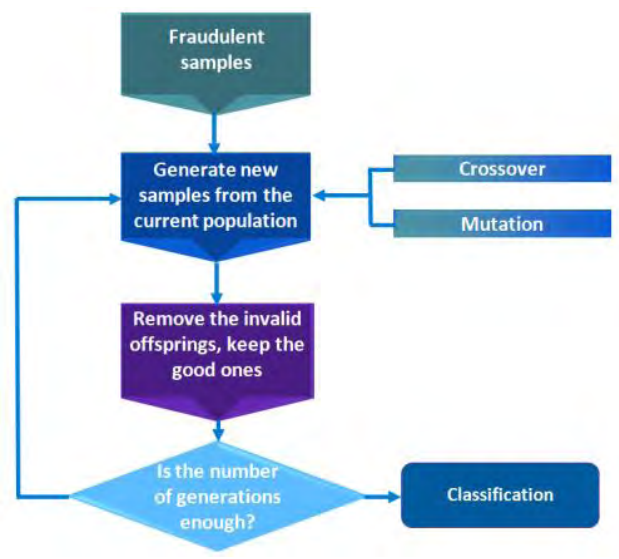

Figure 5. GA Flow

In further details, the genetic algorithm starts with the cluster's samples which act as the parents of the current generation. New synthetic individuals are generated from these samples by the crossover and mutation operators. New individuals will inherit the individual's characteristic of parent's generation, rather than simple duplication. The less fit individuals of this generation are eliminated and the fitter members are selected as the parents for the next generation. This procedure is repeated until a pre-specified number of generations have passed, and the augmented training data set until then is balanced. Thus, binary classifier is more effective in detecting fraudulent transactions.

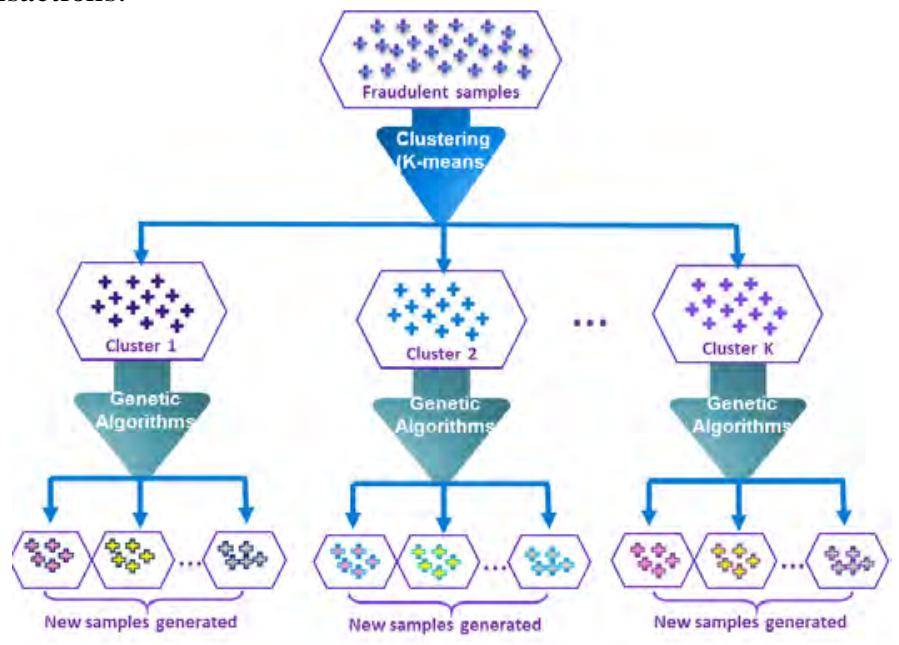

Figure 6. GA generation process

\section{Here are steps of algorithm:}

1. With k=4, fraudulent class $C_{f}$ is divided into four clusters by means of K-means algorithm, the Ith

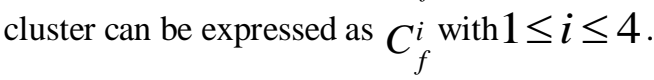

2. The fitness value is calculated in each population $C_{f}^{i}$ and is sorted out. 
3. Carry out random pairing to individual $m_{i}$ inside the $C_{f}^{i}$, the amount of pairing group is $\frac{m_{i}}{2}$.

4. Each pair of individuals of the population $C_{f}^{i}$ can suffer crossover based on a given crossover probability.

5. After crossover, each individual can be mutated, based on a given mutation probability.

This process is repeated the number of times necessary to achieve the desired size of fraudulent population and obtain a balanced training dataset.

\section{EXPERIMENTAL RESULTS}

This section describes the dataset and provides the evaluation metrics used in this work. The results of the experiments of the proposed method are then presented.

\subsection{Dataset Description}

Datasets provide a way to train and verify the efficacy of the proposed techniques and therefore play crucial role for driving the research. One of the difficulties with studying Credit card fraud detection is that considered highly confidential and not disclosed to public (Lopez-Rojas, E. A., and Axelsson, S., 2016; Dal Pozzolo, A. et al, 2014). Hence, researchers have suggested using synthetic data that is modeled after a real data set such that it contains similar patterns. For this work, we use data produced by BankSim, a simulation tool specifically designed to emulate fraud data (Vaughan, G., 2018; Lopez-Rojas, E. A., and Axelsson, S., 2014). The data produced by BankSim is freely available on Kaggle.

BankSim uses a Multi Agent-Based Simulation approach based on a sample of aggregated real transaction data provided by one bank in Spain. The original bank data consists of thousands of records of transactional data from November 2012 to April 2013. BankSim mimics this original bank data using several agents of three different categories: merchants, customers, and fraudsters. These agents interact over a sequence of simulated days, resulting in a log of purchase transactions that closely resemble the original bank data.

The dataset used in this work contains transactions corresponding to card purchases made during 180 simulated days and consists of 594,643 different transactions, among which 7,200 $(\approx 1,2 \%)$ are labeled as "Fraud", while the remaining 587,443 are labeled as "Geniune". Raw data include information about the transactions that are processed. Table 1 describes the feature vector of a transaction message.

Table 1. Feature vectors description

\begin{tabular}{|l|l|}
\hline Name & Description \\
\hline Step & The day the transaction took place from 1 to 180 \\
\hline Customer ID & A number identifying the customer account involved in the transaction \\
\hline Age Category & A categorical value putting the customer into one of 8 different age groups \\
\hline Gender & A categorical variable indicating the gender of the customer \\
\hline Zip Code of account & The zip code associated with the customer \\
\hline Merchant ID & A number identifying the merchant involved in the transaction \\
\hline Zip Code of Merchant & The zip code of the merchant \\
\hline Category purchase & A categorical variable indicating what type of good or service was purchased \\
\hline Amount of purchase & The total amount that the transaction cost \\
\hline Fraud status & A binary variable indicating if the transaction was fraudulent of not \\
\hline
\end{tabular}




\subsection{Performance Metrics}

In order to evaluate our approach, the performance of Random Forest classifier is measured based on accuracy, sensitivity, specificity and precision. These evaluation metrics are used based on their relevance in evaluating imbalanced binary classification problem.

$$
\begin{array}{ll}
\text { Accuracy }=\frac{T P+T N}{T P+F P+T N+F N} & \text { Sensivity }=\frac{T P}{T P+F N} \\
\text { Specificity }=\frac{T N}{F P+T N} & \text { Precision }=\frac{T P}{T P+F P}
\end{array}
$$

True positives (TP) are cases classified as positive which are actually positive. True negative (TN) are cases classified rightly as negative. False positive (FP) are cases classified as positive but are negative cases. False negative (FN) are cases classified as negative but are truly positive. Sensitivity (Recall) gives the accuracy on positive (fraud) cases classification. Specificity gives the accuracy on negative (legitimate) cases classification and precision gives the accuracy in cases classified as fraud (positive).

In this study, $70 \%$ of the dataset is used for training while $30 \%$ is set aside for validating and testing. Accuracy, sensitivity, specificity and precision are used to evaluate the performance of the Random Forest classifier. The accuracy of the classifier for the original imbalanced dataset and balanced dataset by means of application of our proposed approach are presented in Table 2.

Table 2. Accuracy results for balanced and imbalanced set

\begin{tabular}{|l|l|l|}
\hline \multirow{2}{*}{ Metrics } & \multicolumn{2}{|c|}{ RF Classifier } \\
\cline { 2 - 3 } & Imbalanced Set & Balanced Set \\
\hline Accuracy & 0.9537 & 0.9769 \\
\hline Sensitivity & 0.8572 & 0.9514 \\
\hline Specificity & 0.9341 & 0.9696 \\
\hline Precision & 0.7605 & 0.8786 \\
\hline
\end{tabular}

An observation of the metric table shows that there is significant improvement from the balanced dataset distribution for accuracy, sensitivity and specificity of RF classifier. This shows that applying our strategy on a highly imbalanced dataset greatly improves the performance of binary classification.

\section{CONCLUSION}

The credit card fraud detection is becoming important topic of research, as different types of attacks are increasing at an alarming rate. The goal of our work is to deal with the problem of class imbalance and improve fraud detection accuracy on new incoming transactions. Given a training set, an augmented set is produced, containing more examples of the minority class through the generation of mimicked examples by means of genetic operators. While we have presented our strategy in the context of credit card fraud detection, it is quite general and can be extended to other application domains characterized by significant class imbalance rates.

To validate our work, we performed experiments on publicly available credit card fraud detection data, where the minority class is severely underrepresented. Experiments show that a classifier trained on the balanced set outperforms the same classifier trained on the original data, especially as far the sensitivity is concerned, resulting in an effective fraud detection mechanism.

Besides the class imbalance problem, fraud detection algorithms also need to account for the fact that the class conditional distribution changes over time. As future work, we would like to devise a strategy to exploit the temporal information of customer expending behaviors and fraud patterns using Deep Learning architectures. 


\section{REFERENCES}

Aleskerov, E. et al, 1997. CARDWATCH: a neural network based database mining system for credit card fraud detection. In Proceedings of the IEEE/IAFE Computational Intelligence for Financial Engineering (CIFEr). New York City, NY, USA, pp. 220-226.

Ali, A. et al, 2015. Classification with class imbalance problem: A Review. In Int. J. Advance Soft Compu. Appl, Vol. 7, No. 3.

Bahnsen, A. C. et al, 2015. Example-dependent cost-sensitive decision trees. In Expert Systems with Applications, Vol. 42, No. 19,pp 6609-6619.

Bawane, V. S. and Kale, S. M., 2015. Clustering algorithms in MapReduce: a review. In National Conference on Recent Trend in Computer Science and Engineering.

Ben Amor, H. and Rettinger, A., 2005. Intelligent exploration for genetic algorithms: Using self-organizing maps in evolutionary computation. In proceedings of the 2005 conference on Genetic and evolutionary computation, pp 1531-1538.

Bhattacharyya, S. et al, 2011. Data mining for credit card fraud: A comparative study. In Decision Support Syst, Vol. 50, No. 3,pp 602-613.

Breiman, L., 1996. Bagging Predictors. In Journal of Machine Learning, Vol. 24, No. 2,pp 123-140.

Chawla, N. et al, 2002. SMOTE: Synthetic minority over-sampling technique. In Journal of Artificial Intelligence Research, Vol. 16, No. 1,pp 321-357.

Cieslak, D. A. et al, 2012. Hellinger distance decision trees are robust and skew-insensitive. In Data Mining and Knowledge Discovery, Vol. 24, No. 1,pp 136-158.

Dal Pozzolo, A. et al, 2014. Learned lessons in credit card fraud detection from a practitioner perspective. In Expert Systems with Applications, Vol. 41, No. 10,pp 4915-4928.

Dal Pozzolo, A. et al, 2014. Using HDDT to avoid instances propagation in unbalanced and evolving data streams. In Proc. International Joint Conference on Neural Networks (IJCNN). Beijing, China, pp 588-594.

Dal Pozzolo, A. et al, 2015. When is undersampling effective in unbalanced classification tasks?. Machine Learning and Knowledge Discovery in Databases. Cambridge, U.K, pp 200-215.

Freund, Y. and Schapire, R. E., 1996. Experiments with a New Boosting Algorithm. In Proceedings of the Thirteenth International Conference on International Conference on Machine Learning. Bari, Italy, pp. 148-156.

Goldberg, D., 1983. Computer-Aided Gas Pipeline Operation Using Genetic Algorithms And Rule Learning. In University of Michigan, Ann Arbor.

He, H. and Garcia, E. A., 2009. Learning from imbalanced data. In IEEE Transactions on Knowledge and Data Engineering. Vol. 21, No. 9,pp 1263-1284.

Holland, J. H., 1992. Adaptation In Natural And Artificial Systems. In University of Michigan Press.

Jain, A.K, 2010. Data clustering: 50 years beyond k -means. In Pattern Recognition Letters, Vol. 31, No. 8,pp 651-666.

Jha, S. et al, 2012. Employing transaction aggregation strategy to detect credit card fraud. In Expert Systems with Applications, Vol. 39, No. 16,pp 12650-12657.

Krivko, M., 2010. A hybrid model for plastic card fraud detection systems. In Expert Systems with Applications, Vol. 37, No. 8,pp 6070-6076.

Lopez-Rojas, E. A., and Axelsson, S., 2014. BankSim: A bank payment simulation for Fraud detection research. In The 26th European modeling and simulation symposium, pp 144-152.

Lopez-Rojas, E. A., and Axelsson, S., 2016. A review of computer simulation for fraud detection research in financial datasets. In 2016 future technologies conference, pp 932-935.

Sahin, Y. et al, 2013. A cost-sensitive decision tree approach for fraud detection. In Expert Systems with Applications, Vol. 40, No. 15,pp 5916-5923.

Sánchez, D. et al, 2009. Association rules applied to credit card fraud detection. In Expert Systems with Applications, Vol. 36, No. 2,pp 3630-3640.

Sun, Y. et al, 2007. Cost-sensitive boosting for classification of imbalanced data. In Pattern Recognition, Vol. 40, No. 12,pp 3358-3378.

Vaughan, G., 2018. Efficient big data model selection with applications to fraud detection. In International Journal of Forecasting.

Whitrow, C. et al, 2009. Transaction aggregation as a strategy for credit card fraud detection. In Data Mining and Knowledge Discovery. 18, pp. 30-55.

Zhang, J. et al, 2013. A parallel clustering algorithm with mpi-mkmeans. In Journal of Computers, Vol. 8, No. 1. 